\title{
Eletroestimulação neuromuscular na reabilitação da disfagia orofaríngea
}

\author{
Neuromuscular electrical stimulation in the rehabilitation of \\ oropharyngeal dysphagia
}

\author{
Bruno Tavares de Lima Guimarães ${ }^{1}$, Ana Maria Furkim² ${ }^{2}$ Roberta Gonçalves da Silva ${ }^{3}$
}

\begin{abstract}
RESUMO
A reabilitação da disfagia orofaríngea ganhou um novo instrumento terapêutico, a eletroestimulação neuromuscular (EENM), sendo que os mais renomados pesquisadores têm estudado a indicação e os resultados desta abordagem. O objetivo deste trabalho foi apresentar revisão bibliográfica sobre a aplicabilidade da EENM na reabilitação da disfagia orofaríngea. Realizou-se amplo levantamento bibliográfico em bases de dados, englobando as duas últimas décadas de pesquisa na área. Este artigo de revisão mostrou que ainda não há consenso sobre o uso da EENM na reabilitação da disfagia. Constatou-se que a maioria dos trabalhos descreveu o uso da EENM de forma isolada, não relatou as técnicas fonoaudiológicas associadas à eletroterapia e utilizou amostras heterogêneas que agrupavam disfagias orofaríngeas mecânicas e neurogênicas. Somente recentemente programas específicos têm sido delineados e testados em populações mais homogêneas.
\end{abstract}

Descritores: Disfagia/reabilitação; Transtornos de deglutição; Terapia por estimulação elétrica/métodos

\section{INTRODUÇÃO}

A disfagia é uma condição que submete o indivíduo a constante instabilidade clínica, podendo ocasionar complicações como pneumonia aspirativa, desidratação, desnutrição e óbito. Devido a estas questões, este sintoma pode aumentar os custos dos cuidados de saúde com o indivíduo disfágico, bem como prolongar a duração da estada hospitalar ${ }^{(1)}$.

Desta forma, distintas tem sido as propostas para a reabilitação da disfagia orofaríngea pesquisadas ao longo dos anos e discutidas por alguns artigos de revisão $0^{(2-4)}$.

A mais nova abordagem proposta para a reabilitação da disfagia orofaríngea trata-se da eletroestimulação neuromuscular (EENM), considerada uma técnica não invasiva, aplicada através de eletrodos de forma transcutânea. Considerada um dos recursos terapêuticos atuais para a disfagia orofaríngea, a EENM é usada desde 1997 nos Estados Unidos, quando aprovada pelo Food and Drug Administration (FDA), com a finalidade de promover movimentação suprahióidea, laríngea

(1) Fonoaudiólogo clínico - Fortaleza (CE), Brasil.

(2) Doutora, Professora Adjunto do Departamento de Análises Clínicas do Curso de Fonoaudiologia da Universidade Federal de Santa Catarina - UFSC - Florianópolis (SC), Brasil.

(3) Doutora, Professora do Departamento de Fonoaudiologia da Universidade Estadual Paulista Campus de Marília - UNESP - Marília (SP), Brasil.

Endereço para correspondência: Bruno Tavares de Lima Guimarães. R. Joaquim Lima, 585, Papicu, Fortaleza (CE), Brasil, CEP: 60175-005. E-mail: brunotlguimaraes@gmail.com

Recebido em: 28/6/2010; Aceito em: 28/10/2010 e para o favorecimento da contração dos grupos musculares envolvidos diretamente com a deglutiçãa ${ }^{(5-16)}$.

A EENM vem sendo citada como tendo um importante papel em vários segmentos na clínica da reabilitação. A EENM pode ser usada para conseguir o aumento efetivo na força muscular, no tratamento das limitações da amplitude de movimento das articulações devido a restrições de tecidos moles ou fraqueza, para a redução da debilidade no desempenho neuromuscular minimizando a incapacidade associada a espasticidade, para a redução das debilidades de controle do movimento, nos músculos inativos e para favorecer a criação de um feedback que maximize o desempenho muscular após exercícios de contração muscular voluntária ${ }^{(17-21)}$.

O objetivo do presente estudo foi apresentar revisão bibliográfica sobre a aplicabilidade e a eficácia da eletroestimulação neuromuscular na reabilitação da disfagia orofaríngea, a partir de um amplo levantamento bibliográfico nas bases de dados (SciELO, LILACS, PubMed e Cochrane), entre 1983 à 2010, e livros sobre a área.

\section{REVISÃO DE LITERATURA}

A EENM terapêutica foi proposta como uma opção de tratamento para a disfagia orofaríngea, demonstrando benefícios para disfágicos após Acidente Vascular Encefálico (AVE), pós-radioterapia, em pacientes com xerostomia ${ }^{(22)}$, tensões e dores, com resultados favoráveis para o aumento na qualidade vocal e na deglutição desta população ${ }^{(20)}$. No entanto, pouco 
se sabe sobre os efeitos específicos que tem a EENM sobre os aspectos biomecânicos da faringe, da laringe e sobre a deglutição propriamente $\operatorname{dita}^{(12,13,23)}$.

A eletroestimulação transcutânea (TENS) que tem por finalidade a analgesia, foi aplicada com o intuito de reduzir a dor em três pacientes após a cirurgia de câncer de cabeça e pescoço, submetidos a radioterapia, e que apresentavam disfagia. Após a aplicação da TENS todos os pacientes relataram diminuição das dores com redução do uso de analgésicos ${ }^{(5)}$.

A TENS foi aplicada com o objetivo de tratar os efeitos secundários da radioterapia em 13 pacientes que apresentavam dores, xerostomia, mucosite e disfagia. Todos os pacientes apresentaram redução ou ausência dos sintomas iniciais durante a radioterapia e até um ano após o tratamento, com exceção daqueles com recorrência da doença. Não foram relatados efeitos secundários ${ }^{(22)}$.

No final de 2001, a fim de obterem a habilitação junto ao $\mathrm{FDA}^{(9)}$ para a liberação de um aparelho eletroestimulador, o VitalStim ${ }^{\circledR}$, de uso especifico para o tratamento da disfagia, pesquisadores apresentaram um amplo estudo sobre a utilização da EENM no tratamento da disfagia, desenvolvido entre 1997 e 2000, demonstrando ser a EENM eficiente e segura para esta modalidade terapêutica. Os estudos foram monitorados e revisados com 892 pacientes, em diversas faixas etárias, de ambos os gêneros e com diversos diagnósticos, dos quais 58\% apresentavam disfagia grave. Os pacientes foram estimulados em três condições de terapia: sensorial (E1), motor (E2) e com estimulação térmica (ET). Os primeiros 157 pacientes foram randomizados para grupos E1 e ET, sendo os 735 restantes distribuídos em qualquer um dos grupos E1 e E2. Os pacientes foram tratados até atingirem deglutição funcional ou até quando não houvesse mais possibilidades de mudanças benéficas aos indivíduos. Os pacientes dos grupos E1 e E2 somaram $98,4 \%$ de sucesso em comparação com $32,7 \%$ do grupo ET. Nos pacientes que apresentaram disfagia grave e que foram atendidos com EENM, o índice de eficácia chegou a $97,5 \%{ }^{(10,11)}$. Utilizando-se o equipamento da VitalStim ${ }^{\circledR}$, os autores compararam os efeitos da EENM no tratamento da disfagia com a estimulação térmica fria. Foram selecionados 99 pacientes, todos disfágicos e com evidências de aspiração laringotraqueal. Destes, 63 pacientes foram tratados com EENM enquanto 36 pacientes, apenas com ET. Nos dois grupos o objetivo principal foi recuperar a capacidade de ingestão de alimentos por via oral. Dos pacientes tratados com EENM, 98\% (62/63) obtiveram mudanças benéficas na deglutição, contra $42 \%$ do grupo da ET $(15 / 36)^{(11)}$.

No Brasil, o uso da eletrototerapia já foi relatado em forma de eletroestimulação transcutânea (TENS) para fins de relaxamento laríngeo e de analgesia em pacientes portadores de disfonia, desconforto laríngeo e cervical,-sendo aplicado para reabilitação dos quadros de disfonia orgânica e funcional ${ }^{(24,25)}$.

A EENM foi usada em um grupo de 23 pacientes com quadro de disfagia moderada e grave, sendo 11 homens e 12 mulheres, com idades variando entre 37 e 87 anos, com capacidade de elevação laríngea reduzida e que não apresentaram bons resultados com a terapia convencional para disfagia. Foi aplicada a EENM em sessões diárias de até quatro horas de estimulação contínua e 21 casos chegaram até ao final do programa. O resultado foi considerado satisfatório para todos os pacientes que deglutiram $3 \mathrm{ml}$ de água sem penetração ou aspiração laringotraqueal. Os autores concluíram que a EENM pode ser um recurso útil para pessoas com disfagia moderada, por ser uma técnica não invasiva e por possibilitar maior facilitação da deglutição, já que as contrações provocadas pela EENM aumentam a excursão laríngea durante a deglutição. A EENM reduz o tempo de uso da sonda nasogástrica e da gastrostomia, bem como o tempo de internação hospitalar ${ }^{(26)}$.

O uso da EENM foi relatado em um grupo de 30 crianças com disfagia (múltipla etiologia) que já vinham recebendo terapia convencional, porém com baixo índice de eficácia. Os pacientes receberam uma hora direta de EENM até a recuperação parcial ou total da deglutição, com média de 22 dias de eletroterapia. Dessas 30 crianças, 17 obtiveram mudanças benéficas na deglutição enquanto cinco delas recuperaram $100 \%$. Outro dado importante relatado foi a ausência de alterações sobre a pressão arterial e de laringoespasmos e bradicardia ${ }^{(1)}$.

Outro estudo ${ }^{(6)}$ com EENM envolveu 20 pacientes com disfagia, sendo dez com sequelas de AVE, quatro com problemas respiratórios, dois com disfunção cricofaríngea, três com câncer de cabeça e pescoço e um com miopatia esteroide. Todos foram submetidos a EENM durante dez sessões de tratamento, observando-se resultados positivos, entre a quinta e a nona sessões, no trânsito faríngeo, na força de injeção do bolo e na redução de resíduos em valécula e seio piriforme durante a deglutição.

Por meio de estudo randomizado foram avaliados os efeitos da aplicação de EENM em 12 pacientes com xerostomia que realizaram radioterapia após cirurgia de cabeça e pescoço. Os pacientes foram tratados com EENM em média por dez sessões. Destes, $67 \%$ dos pacientes relataram mudanças significativas na produção de saliva, passando a necessitar de menos líquido durante as refeições para conseguirem realizar uma deglutição mais funcional, além de maior umidade na língua e lábios ${ }^{(19)}$.

A partir de um estudo retrospectivo foram analisados os resultados da aplicação da EENM em 80 pacientes com disfagia. Destes, 40 pacientes realizaram tratamento com EENM e 40 pacientes foram submetidos à terapia tradicional para disfagia. A etiologia da disfagia incluía insuficiência respiratória, AVE e outras condições crônicas. A terapia tradicional consistiu na combinação de exercício terapêutico, alterações na dieta e/ou consistência e de manobras voluntárias de deglutição. A EENM foi administrada a uma frequencia de $80 \mathrm{~Hz}$ e a intensidade ideal foi determinada quando se identificava uma resposta motora visualmente ou verbalmente. Foi usada escala de grau de comprometimento para disfagia orofaríngea pré e pós-fonoterapia. Os resultados indicaram que ambos os grupos mostraram mudanças benéficas na deglutição após o tratamento. Os pacientes que se submeteram a EENM demonstraram mais resultados positivos do que o grupo de terapia tradicional nos aspectos relacionados à biomecânica da deglutição. Além disso, os indivíduos que receberam EENM necessitaram de menor tempo de internação do que aqueles que receberam terapia tradicional de deglutição ${ }^{(27)}$.

A aplicação da EENM com os eletrodos fixados sobre a região submandibular e laríngea foi realizada em 29 adultos sau- 
dáveis sem transtornos de deglutição (14 do gênero masculino e 15 do gênero feminino). Os voluntários foram avaliados em movimento, parados e deglutindo. Tanto a frequência quanto a intensidade dos estímulos estavam de acordo com os sugeridos pelo VitalStim ${ }^{\circledR}$. Para a avaliação foram utilizados os protocolos da VitalStim ${ }^{\circledR}$, videofluoroscopia da deglutição, escala de segurança para deglutição, além das medidas biomecânicas da laringe e do hioide. Os autores ${ }^{(13)}$ observaram que utilizando-se dois pares de eletrodos em ambas as regiões submandibular e laríngea, puderam ser ativados simultaneamente músculos opostos. Além disso, observaram significativa descida do osso hioide e da laringe durante o repouso, atribuindo tal resultado à contração dos músculos esternohióideo e omohióideo. Em todas as posições onde foram fixados os eletrodos observou-se redução na elevação do osso hioide e reduções significativas da elevação da laringe em todas as modalidades de deglutição, sendo que o uso da EENM com dois ou mais pares de eletrodos pode ser prejudicial para a deglutição.

Outros autores compararam os efeitos da EENM com os efeitos da terapia tradicional para disfagia, a partir da análise de 22 pacientes submetidos inicialmente a videofluoroscopia e que apresentavam diferentes etiologias para disfagia. Os participantes foram divididos em dois grupos, sendo um experimental que recebeu EENM (11 sujeitos) e um controle que recebeu terapia tradicional (11 sujeitos). O grupo experimental recebeu entre 15 e 45 minutos de terapia tradicional com exercícios, manobras, diferentes consistências de alimento e estimulação neuromuscular profunda na faringe, sendo esses procedimentos baseados nos achados videofluoroscópicos. No grupo experimental, a EENM estava de acordo com o manual do VitalStim®. Os autores não observaram diferenças entre a terapia tradicional e a terapia com EENM. Consideraram importante a continuidade de estudos futuros que analisem melhor as variáveis da amostra, tendo em vista o alto custo hospitalar ou para os serviços de home-care ocasionados pelos quadros disfágicos ${ }^{(14)}$.

A EENM foi usada com o objetivo de verificar o aumento da atividade e da amplitude muscular submandibular. Os participantes não apresentavam transtorno de deglutição e foram estimulados com EENM por cinco dias consecutivos, passando dois dias sem estimulação elétrica, voltando a receber cinco dias seguidos de EENM. Destes, sete dos participantes não apresentaram aumento significativo na atividade mioelástica, dois exibiram hipersensibilidade na região estimulada e um apresentou grande diminuição na atividade da musculatura submandibular. Os autores teorizaram que cada resposta à estimulação elétrica pode ser especifica para cada frequência. Uma participante apresentou grandes ganhos na atividade muscular após duas semanas de tratamento, sendo este dado atribuído ao aumento da intensidade da estimulação durante os dias de estimulação. Os autores concluíram que a EENM passiva nos músculos submandibulares para maximizar a fase faríngea da deglutição não esta bem definida ${ }^{(28)}$.

Foi aplicada EENM em 18 pacientes (12 homens e seis mulheres), todos em regime de internação hospitalar ou atendimento ambulatorial, com disfagia de causas distintas, sendo que cinco apresentavam disfagia grave. A EENM foi administrada de acordo com o manual de instruções do fabricante
VitalStim e o número total de sessões prestado aos pacientes variou de sete a 28 sessões. Nestes 18 sujeitos, constatou-se retirada de via alternativa em seis (33\%), algum tipo de evolução em $11(61 \%)$ e nove $(50 \%)$ demonstraram resultados estatisticamente significantes. Apenas dois dos cinco pacientes com disfagia grave apresentaram algum tipo de mudança; entretanto, nenhum deles foi capaz de interromper a alimentação enteral após a EENM. Os autores concluíram que a terapia com EENM parece ser benéfica somente para os pacientes com grau leve ou moderado de disfagia. Pacientes com disfagia grave demonstraram pouca ou nenhuma mudança benéfica para a deglutição. Os autores sustentaram que a EENM pode ser importante junto com a terapia tradicional ${ }^{(16)}$.

O efeito da EENM sobre a deglutição e a verificação sobre se mudanças ocorridas vinham acompanhadas por reorganização cortical foram avaliados em oito pacientes disfágicos. Estes pacientes foram tratados com EENM seguindo um protocolo de uso para eletroterapia, sendo que dos oito sujeitos, quatro pacientes apresentavam disfagia por comprometimento cortical e quatro por comprometimento do neurônio motor inferior. Os pacientes receberam tratamento cinco dias por semana durante duas semanas, uma hora por dia. Foram obtidos resultados positivos e significativos na capacidade de deglutir, com aumento significativo na representação e na excitabilidade cortical. Os autores correlacionaram a evolução na função da deglutição com a reorganização cortical, sugerindo uma relação causal entre ambas ${ }^{(29)}$.

Os efeitos fisiológicos da EENM sobre a eficácia da deglutição foram avaliados em 11 pacientes disfágicos, com distintas etiologias, randomizados para receberem EENM em nível sensorial ou motor. Foi avaliada a movimentação do osso hioide e da laringe durante a EENM máxima e em condição de repouso; além disso, foi utilizada uma escala de avaliação da penetração laríngea e da aspiração laringotraqueal, especialmente criada para este estudo. $\mathrm{O}$ osso hioide apresentou descida máxima durante a EENM, resultando em aumento na aproximação hiolaríngea. Os autores não encontraram alterações significativas durante as deglutições, quer na estimulação em nível sensorial quer na estimulação motora ${ }^{(30)}$.

O conhecimento dos terapeutas sobre o uso da EENM e sobre o conhecimento prático para o tratamento de pacientes disfágicos já foi pesquisado. Foram enviados 2000 questionários, aos quais 840 terapeutas $(70 \%)$ responderam que usavam a técnica com o tempo das sessões durando, em média, uma hora, de três a cinco dias da semana. Destes, $90 \%$ utilizavam a EENM junto com outras técnicas tradicionais e a maioria observou avanços na mudança da dieta dos pacientes e diminuição na penetração e na aspiração em mais de $50 \%$ dos pacientes. O nível de satisfação comunicado pelos pacientes aos terapeutas sobre o uso da EENM durante a terapia foi de $80 \%$ e entre os profissionais foi de $78 \%{ }^{(31)}$.

Outro estudo, realizado com seis pacientes adultos portadores de disfagia orofaríngea, aplicou, durante 15 sessões, um protocolo padronizado de exercícios para deglutição junto com $\mathrm{EENM}^{(32)}$. Os mesmos foram reavaliados ao término das 15 sessões e após seis meses, utilizando parâmetros como o nível de ingestão oral de alimentos, a mudança no peso corporal, a percepção do paciente para a capacidade de deglutição e quanto 
às alterações dos aspectos da biomecânica da deglutição. Os resultados obtidos indicaram mudança positiva na deglutição funcional, aumento do nível de ingestão oral, ganho de peso, maior percepção do paciente sobre a capacidade de deglutição, restabelecimento eficiente na movimentação do osso hioide e na elevação laríngea durante a deglutição. Nenhum paciente experimentou complicações relacionadas a laringoespasmos e alterações na pressão arterial. Os autores concluíram que a terapia sistemática para a disfagia orofaríngea crônica usando-se EENM como coadjuvante é eficaz para a capacidade funcional da deglutição, gerando inclusive ganho de peso.

O benefício da EENM para casos de disfagia causada por AVE vem sendo investigado ${ }^{(33)}$. Dez pacientes com disfagia crônica foram incluídos no estudo, sendo divididos em dois grupos. Aplicou-se a EENM com uma intensidade máxima tolerável nos músculos digástrico e tirohióideo por uma hora diária, cinco dias por semana, durante quatro semanas, em um dos grupos. Os pacientes do outro grupo receberam EENM na mesma condição, mas com intensidade diferente. Os mesmos foram avaliados com uma escala para mensurar o nível de ingestão oral e análise cinemática do movimento do osso hioide antes do tratamento, duas semanas mais tarde, ou seja, durante o tratamento e quatro semanas após, quando o tratamento havia sido finalizado. $\mathrm{O}$ grau de comprometimento da disfagia diminuiu em ambos os grupos, sendo que a diferença não foi estatisticamente significativa. Mesmo assim, os autores concluíram que a EENM terapêutica com o máximo de intensidade tolerável sobre os músculos digástrico e tirohióideo pode ser eficaz em pacientes com disfagia crônica decorrente de AVE.

Um estudo randomizado para avaliar e comparar o resultado da EENM com a terapia tradicional (TT) para disfagia em pacientes com AVE foi realizado em três centros europeus de tratamento da disfagia ${ }^{(34)}$. Participaram deste estudo 25 pacientes (16 homens e nove mulheres) entre 50 e 80 anos, dos quais 12 foram randomizados para EENM e 13 para o TT. Todos apresentaram AVE há mais de três meses antes do estudo, sendo o AVE hemisférico, sem envolvimento do tronco encefálico, com capacidade de comunicação e disfagia pré e pós-fonoterapia foram realizadas medições videoradiográficas, avaliação da deglutição, estado nutricional, teste motor oral funcional e a escala visual analógica (EVA) para a auto-avaliação. Um grupo recebeu EENM sem qualquer outra técnica ou manobra terapêutica associada e o outro grupo foi tratado com uma combinação de técnicas e exercícios tradicionais. Todos os indivíduos receberam 15 sessões de terapia, com duração de uma hora durante três semanas. Estatisticamente foram encontrados efeitos positivos para ambos os grupos nos quesitos capacidade e segurança para deglutir, mas não houve diferenças estatisticamente significantes no efeito da terapia entre os grupos. As correlações entre as medições foram consideradas baixas e a percepção do paciente sobre as mudanças benéficas em sua deglutição tiveram baixa correlação com os resultados a partir do objetivo da avaliação.

Em outro estudo, 25 pacientes com esclerose múltipla e disfágicos, sendo 16 do gênero masculino e nove do gênero feminino, com média de idade de 53,1 anos foram avaliados após o uso de $\operatorname{EENM}^{(7)}$. Os mesmos receberam o tratamento dois dias por semana, durante três semanas, sendo instruídos a deglutir no exato momento da percepção do disparo do estímulo da EENM. Foram utilizados como parâmetros pré e pós-fonoterapia o desempenho da excursão hiolaríngea, cronometrado o tempo de deglutição para diversas consistências, a pontuação na escala de segurança para deglutição penetração/aspiração, o custo benefício e a qualidade de vida antes e após o tratamento com EENM. Os autores relataram evolução significativa na qualidade da deglutição para líquidos finos, com redução na estase em seios piriformes, na penetração e na aspiração e aumento na proteção das vias aéreas inferiores.

Foi relatado o caso de um homem com 76 anos, portador da síndrome opercular, caracterizada pela perda bilateral completa do controle voluntário da face, língua, faringe, músculos mastigatórios e grave disfagia ${ }^{(35)}$. Após o tratamento fonoaudiológico tradicional para disfagia, não houve resultados positivos quanto a deglutição. Posteriormente, o paciente recebeu tratamento com EENM por cinco meses, com dois pares de eletrodos, em sessões de uma hora, cinco dias consecutivos por semana, associado à terapia tradicional. Os principais objetivos do tratamento foram aumentar a elevação hiolaríngea e estimular a aferência sensorial para facilitar o aparecimento da resposta faríngea da deglutição. Inicialmente pequenas quantidades (2 até $5 \mathrm{ml}$ ) de líquido espessado foram oferecidas ao paciente e progressivamente passou-se à ingestão de alimentos de diferentes consistências, utilizando manobras deglutição. Com o término do tratamento, o indivíduo voltou para a alimentação por via oral com pequenas restrições na dieta, composta de várias consistências e exigindo preparação especial. Um ano após a terapia, a condição clínica do paciente não mudou, e este se encontra com dieta oral e sem pneumonia.

Um grupo de pacientes pós-radioterapia para câncer da nasofaringe e com disfagia recebeu EENM, enquanto um segundo grupo recebeu terapia tradicional ${ }^{(36)}$. As mudanças benéficas foram maiores no grupo que utilizou EENM, porém os autores advertem para a necessidade de novos estudos.

Foram testados os efeitos da EENM sobre a região de elevação do osso hioide durante a deglutição em 16 indivíduos voluntários saudáveis, divididos em dois grupos ${ }^{(15)}$. Nos indivíduos do grupo experimental os eletrodos foram fixados acima do músculo infrahióideo e foi solicitado aos mesmos que deglutissem com esforço, de modo a provocar uma excursão hiolaríngea no exato instante da percepção da EENM. A mesma condição de deglutição foi solicitada ao grupo controle, sendo a EENM ajustada um pouco acima do limiar sensorial. Ambos os grupos receberam entre dez e 20 minutos de estímulos ao longo de duas semanas. Foi registrada atividade elétrica nos músculos submandibulares e a movimentação hióidea em três marcações (pré, imediatamente após a sessão e duas semanas após as aplicações), sendo que no grupo experimental a movimentação do eixo do hioide foi observada duas semanas após as aplicações e a atividade mioelétrica não foi afetada em nenhuma das condições avaliadas. Os autores concluíram que a deglutição com esforço, associada à EENM, aumenta o grau de elevação do hioide de voluntários saudáveis, precisando ser testada em pacientes disfágicos.

Foi aplicada a EENM sobre a região submentual em 11 pacientes com disfagia pós-AVE ${ }^{(37)}$. Todos os pacientes apresentavam aspiração laringotraqueal, estase alimentar e salivar. 
Receberam EENM por uma hora por dia durante cinco dias (frequência de $80 \mathrm{~Hz}$, no limiar motor). Durante a estimulação elétrica, os pacientes foram convidados a deglutir uma medida de colher de chá da consistência pastosa ou líquida. A deglutição foi avaliada antes e depois da semana de estímulos, utilizando um questionário de índice de desvantagem para disfagia, videofluoroscopia e mapeamento cortical dos músculos da faringe. Os resultados do questionário mostraram que os sintomas de disfagia orofaríngea diminuíram, enquanto a videofluoroscopia mostrou redução dos resíduos faríngeos, diminuição no tempo de reação para deglutição, maior eficiência no trânsito orofaríngeo, no tempo do trânsito faríngeo e maior duração do fechamento da laringe. Para os autores, estes resultados indicaram que os estímulos elétricos sobre a área submentual podem contribuir para a reabilitação da disfagia pós-AVE, otimizando a coordenação da deglutição.

O primeiro estudo brasileiro de revisão sobre o uso da EENM na disfagia orofaríngea realizou levantamento bibliográfico de 1996 a $2007^{(38)}$. Os autores concluíram que a eficácia da reabilitação na disfagia orofaríngea com uso de EENM varia de 70 a $80 \%$. Ressaltaram que esta é uma técnica nãoinvasiva e de fácil aplicação, devendo, portanto, ser explorada juntamente com o profissional da Fonoaudiologia. Concluíram ainda que os estudos recentes usaram amostras heterogêneas, dificultando a compreensão sobre a eficácia desta técnica, uma vez que para este controle é necessária uma amostra homogênea na qual as manifestações e o grau de comprometimento pelas disfagias seja similar. Outra questão levantada pelos autores foi a de que, nos estudos analisados, não há descrição detalhada dos procedimentos terapêuticos utilizados e de sua frequência; além disso, há pouca valorização dos achados na deglutição funcional.

A eficácia da EENM foi avaliada de forma randomizada em 36 pacientes disfágicos pós-AVE ${ }^{(39)}$, distribuídos em dois grupos. No grupo experimental foram aplicadas a EENM e a estimulação térmica simultaneamente, enquanto no grupo controle apenas a estimulação térmica. A deglutição foi avaliada quatro semanas após a terapia por meio de uma escala para identificar a penetração e a aspiração laringotraqueal. Além disso, pontuaram também os itens desconforto e grau de satisfação dos pacientes. Dos 36 pacientes, 28 completaram o estudo, sendo 16 do grupo experimental e 12 do grupo controle. Os dois grupos apresentaram mudanças positivas, porém o grupo experimental mostrou uma evolução mais favorável no parâmetro biomecânica da deglutição, com diminuição da penetração/aspiração e resultados positivos no trânsito faríngeo. Os autores concluíram que a EENM e a estimulação térmica associadas são eficientes para o tratamento da disfagia pós-AVE.

\section{DISCUSSÃO}

A revisão de literatura nesta temática evidenciou claramente a ênfase que vem sendo dada, nos Estados Unidos e Europa, para a realização de estudos randomizados que possam promover evidências sobre a aplicabilidade e a eficácia de uma nova abordagem terapêutica em disfagia orofaríngea. No Brasil encontramos um único $\operatorname{artigo~}^{(38)}$, também de revisão, o que pode sugerir que ainda necessitamos tanto de muito investimento na formação prática do profissional que atua com disfagia orofaríngea, quanto de normas que regulem esta prática.

Os estudos, até o presente momento, não apresentam consenso sobre este método, não sendo diferente de qualquer outra área quando a temática ainda é recente, porém apontam questões de fundamental relevância para os critérios de elegibilidade dos pacientes, bem como para a definição do método.

Parece claro, após esta revisão, que há diferenças tanto na aplicabilidade quanto na eficácia deste método quando usado para populações com disfagia mecânica e neurogênica, sendo ainda necessário esclarecer dentre estas, qual a etiologia das mais beneficiadas ${ }^{(34,36)}$. Assim, confirmando a tendência dos estudos de eficácia em disfagia e independente dos métodos terapêuticos aplicados, há de se considerar a especificidade das etiologias, caso queiramos comprovar a atuação de práticas fonoaudiológicas.

\section{COMENTÁRIOS FINAIS}

O uso da EENM na reabilitação da disfagia orofaríngea, bem como a eficácia encontrada na reabilitação das distintas populações disfágicas, ainda merece investigação. As mais complexas questões identificadas em nosso estudo de revisão, assim como em todos os trabalhos iniciais com outras técnicas propostas para a reabilitação da disfagia, tratam da heterogeneidade das amostras encontradas nas pesquisas, da ausência de um protocolo universal tanto para controle da eficácia da reabilitação como para padronizar a aplicação da EENM. Portanto, é compreensível que ainda não tenhamos clareza sobre a eficácia deste procedimento sobre distintos parâmetros. Constatamos, no entanto, que os estudos atuais, também preocupados com esta questão, estão delineando pesquisas com amostras mais homogêneas, sendo tal prática mais frequente na população com AVE. Além disto, as pesquisas elegeram parâmetros como a biomecânica, o grau de comprometimento da disfagia, o nível de ingestão oral, a autopercepção e a qualidade de vida como itens importantes a serem mensurados pré e pós-fonoterapia com EENM.

Desta forma, é importante que saibamos que até o presente, as pesquisas apontaram mudanças positivas após a aplicação da EENM, demonstrando possibilidade para o aumento da movimentação hiolaríngea, diminuição no tempo de trânsito faríngeo, redução da xerostomia nos casos de radioterapia e aumento do nível de ingestão oral.

Por fim, sugerimos que a área de reabilitação na disfagia orofaríngea considere a eletroterapia como um tratamento possível e parceiro dos tratamentos tradicionais, podendo ser mais um recurso para aumentar a eficácia da reabilitação nos quadros disfágicos. 


\begin{abstract}
The rehabilitation of oropharyngeal dysphagia has a new therapeutic tool, the neuromuscular electrical stimulation (NMES), and the most renowned researchers have been studying the applicability and the results of this approach. The aim of this study was to present a literature review regarding the applicability of NMES in the rehabilitation of oropharyngeal dysphagia. An extensive literature review was carried out, considering the last two decades of research in the area. The review showed that there is still no consensus on the use of NMES in the rehabilitation of dysphagia. It was found that most studies described the use of NMES in isolation, did not describe the techniques associated with speech-language therapy associated to electrotherapy, and used heterogeneous samples that clustered mechanical and neurogenic oropharyngeal dysphagia. Only recently specific programs have been designed and tested in more homogeneous populations.
\end{abstract}

Keywords: Dysphagia/rehabilitation; Deglutition disorders; Electric stimulation therapy/methods;

\title{
REFERÊNCIAS
}

1. Christiaanse M, Glynn J, Bradshaw J. Experience with transcutaneous electrical stimulation: A new treatment option for the management of pediatric dysphagia. NCSHA. Charleston; 2003.

2. Silva RG. A eficácia da reabilitação em disfagia orofaríngea. Pró-Fono. 2007;19(1):123-30.

3. Furkim AM, Sacco ABF. Eficácia da fonoterapia em disfagia neurogênica usando a escala funcional de ingestão por via oral (FOIS) como marcador. Rev CEFAC. 2008;10(4):503-12.

4. Cola PC, Gatto AR, Silva RG, Schelp AO, Henry MACA. Reabilitação em disfagia orofaríngea neurogênica: sabor azedo e temperatura fria. Rev CEFAC. 2008;10(2):200-5.

5. Bauer W. Electrical treatment of severe head and neck cancer pain. Arch Otolaryngol. 1983;109(6):382-3.

6. Belafsky P, Speirs J, Hiss S, Postma G. The safety and efficacy of transcutaneous electrical stimulation in treating dysphagia: preliminary experience. In: Program and abstracts of the Southern Section of the American Laryngological, Rhinological and Otological Society, Inc; Jan 8-11, 2004; Marco Island, Fla. [Abstract 9].

7. Bogaardt H, van Dam D, Wever NM, Bruggeman CE, Koops J, Fokkens WJ. Use of neuromuscular electrostimulation in the treatment of dysphagia in patients with multiple sclerosis. Ann Otol Rhinol Laryngol. 2009;118(4)241-6.

8. Logemann JA. The effects of VitalStim on clinical and research thinking in dysphagia. Dysphagia. 2007;22(1):11-2.

9. Food and Drug Administration - FDA. VitalStim 510(k) clearance document K023347; 2002.

10. Freed ML, Freed L, Chatburn RL, and Christian M. Electrical stimulation for swallowing disorders caused by stroke. Respir Care. 2001;46(5):466-74.

11. Freed ML. Clinical trial data in support of VitalStim 510(k) clearance application with FDA; 2001.

12. Carnaby-Mann GD, Crary MA. Examining the evidence on neuromuscular electrical stimulation for swallowing: a meta-analysis. Arch Otolaryngol Head Neck Surg. 2007;133(6):564-71.

13. Humbert IA, Poletto CJ, Saxon KG, Kearney PR, Crujido L, Wright-Harp W, et al. The effect of surface electrical stimulation on hyolaryngeal movement in normal individuals at rest and during swallowing. J Appl Physiol. 2006;101(6):1657-63.

14. Kiger M, Brown CS, Watkins L. Dysphagia management: an analysis of patient outcomes using VitalStim therapy compared to traditional swallow therapy. Dysphagia. 2006;21(4):243-53.

15. Park JW, Oh JC, Lee HJ, Park SJ, Yoon TS, Kwon BS. Effortful swallowing training coupled with electrical stimulation leads to an increase in hyoid elevation during swallowing. Dysphagia. 2009;24(3):296-301.

16. Shaw GY, Sechtem PR, Searl J, Keller K, Rawi TA, Dowdy E. Transcutaneous neuromuscular electrical stimulation (VitalStim) curative therapy for severe dysphagia: myth or reality? Ann Otol Rhinol Laryngol. 2007;116(1):36-44.

17. Guirro E, Guirro R, editores. Fisioterapia Dermato-funcional: fundamentos, recursos e patologias. 3a ed. São Paulo: Manole; 2002.

18. Evangelista AR, Gravina GA, Borges FS, Vilardi Júnior NP. Adaptação da característica fisiológica da fibra muscular por meio de eletroestimulação. Fisioter Bras. 2003;4(5):326-34.

19. McDuffie CM, Morgan M, Armstrong C, Nathan CA. Electrical stimulation of post irradiated head and neck SCCA. Otolaryngol Head Neck Surg. 2005;133(2 Suppl): 101.

20. Pena R, Barbosa LA, Ishikawa NM. Estimulação elétrica transcutânea do nervo (TENS) na dor oncológica: uma revisão da literatura. Rev Bras Cancerol. 2008;54(2):193-9.

21. Ptok M, Strack D. Electrical stimulation-supported voice exercises are superior to voice exercise therapy alone in patients with unilateral recurrent laryngeal nerve paresis: results from a prospective, randomized clinical trial. Muscle Nerve. 2008;38(2):1005-11.

22. Boswell NS. Neuroelectric therapy eliminates xerostomia during radiotherapy: a case study. Am J Electromed, 1989;115:105-7.

23. Doeltgen S, Huckabee M, Dalrymple-Alford J, Ridding M, O'Beirne G. Effects of event-related electrical stimulation on motor evoked potentials at the submental muscle group. Dysphagia. 2008;23(4):436.

24. Guimarães BT. A eletroestimulação nervosa transcutânea no relaxamento laríngeo. Rev Lugar Fonoaudiol (Estácio de Sá). 1992;3: 27-34.

25. Guimarães, BTL. Relaxamento laríngeo com o uso da eletroestimulação nervosa transcutânea (TENS): um estudo comparativo. Rev Fonoaudiol Brasil. 2001; 1(1):20-8. Disponível em: http://www.fonoaudiologia.org. br/publicacoes/rev-set01-n4.pdf

26. Leelamanit V, Limsakul C, Geater A. Synchronized electrical stimulation in treating pharyngeal dysphagia. Laryngoscope. 2002;112(12):2204-10.

27. Blumenfeld L, Hahn Y, Lepage A, Leonard R, Belafsky PC. Transcutaneous electrical stimulation versus traditional dysphagia therapy: a nonconcurrent cohort study. Otolaryngol Head Neck Surg. 2006;135(5):754-7.

28. Suiter DM, Leder SB, Ruark JL. Effects of neuromuscular electrical stimulation on submental muscle activity. Dysphagia. 2006;21(1):56-60.

29. Oh BM, Kim DY, Paik NJ. Recovery of swallowing function is accompanied by the expansion of the cortical map. Int $\mathrm{J}$ Neurosci. 2007;117(9):1215-27.

30. Ludlow CL, Humbert I, Saxon K, Poletto C, Sonies B, Crujido L. Effects of surface electrical stimulation both at rest and during swallowing in chronic pharyngeal dysphagia. Dysphagia. 2007;22(1):1-10.

31. Crary MA, Carnaby-Mann GD, Faunce A. Electrical stimulation therapy for dysphagia: descriptive results of two surveys. Dysphagia. 2007;22(3):165-73.

32. Carnaby-Mann GD, Crary MA. Adjunctive neuromuscular electrical 
stimulation for treatment-refractory dysphagia. Ann Otol Rhinol Laryngol. 2008;117(4):279-87.

33. Kim DY, Koh ES, Kang BS, Han TR, Lee SU. Effects of electrical stimulation for dysphagia caused by stroke. J Korean Acad Rehabil Med. 2008;32(1):9-14.

34. Bülow M, Speyer R, Baijens L, Woisard V, Ekberg O. Neuromuscular electrical stimulation (NMES) in stroke patients with oral and pharyngeal dysfunction. Dysphagia. 2008;23(3):302-9.

35. Baijens LW, Speyer R, Roodenburg N, Manni J. The effects of neuromuscular electrical stimulation for dysphagia in opercular syndrome: a case study. Eur Arch Otorhinolaryngol. 2008;265(7):82530 .
36. Lin PH, Hsiao TY, Chang YC, Ting LL, Chen WS, Chen SC, Wang TG. Effects of functional electrical stimulation on dysphagia caused by radiation therapy in patients with nasopharyngeal carcinoma. Support Care Cancer. 2009 Nov 29. [Epud ahead of print].

37. Gallas S, Marie JP, Leroi AM, Verin E. Sensory transcutaneous electrical stimulation improves post-stroke dysphagic patients. Dysphagia. 2009 Oct 24. [Epud ahead of print].

38. Soares TMC, Conceição TMA, Cardoso F, Beresford H. Avaliação da estimulação elétrica no tratamento da disfagia secundário ao acidente vascular encefálico. Acta Fisiátrica. 2009;16(4):191-5.

39. Lim KB, Lee HJ, Lim SS, Choi YI. Neuromuscular electrical and thermal-tactile stimulation for dysphagia caused by stroke: a randomized controlled trial. J Rehabil Med. 2009;41(3):174-8. 